Systematic review with meta analysis

\section{With statin co-administration, drugs designed to increase HDL have no impact on cardiovascular outcomes}

10.1136/ebmed-2014-110084

\section{Paul N Durrington}

Cardiovascular Research Group, University of Manchester, Manchester, UK

Correspondence to: Professor Paul N Durrington, Cardiovascular Research Group, University of Manchester, Core Technology Facility, 46 Grafton Street, Manchester M13 9NT, UK; pdurrington@manchester.ac.uk

Commentary on: Keene D, Price C, Shun-Shin MJ, et al. Effect on cardiovascular risk of high density lipoprotein targeted drug treatments niacin, fibrates, and CETP inhibitors: meta-analysis of randomised controlled trials including 117,411 patients. BMJ 2014;349:g4379.

\section{Context}

Decreasing low-density lipoprotein (LDL) cholesterol with statin treatment reduces cardiovascular disease (CVD) incidence. The National Institute for Health and Care Excellence (NICE) has recently recommended that firstline statin treatment in primary prevention should be atorvastatin $20 \mathrm{mg}$ daily and in secondary prevention atorvastatin $80 \mathrm{mg}$ daily, doses which typically decrease LDL cholesterol by $43 \%$ and 55\%, respectively. ${ }^{1}$ In many patients this will leave little scope to reduce residual CVD risk by additional LDL lowering. Attention is therefore drawn to the epidemiological inverse association between HDL cholesterol and CVD risk. This is widely exploited in the prediction of CVD risk, but is HDL cholesterol also 'good cholesterol' in the sense that raising its level therapeutically can prove beneficial? Three lipid-modifying drug classes are known to raise HDL: niacin (nicotinic acid), fibrates and cholesteryl ester transfer protein inhibitors (CETPi's).

\section{Methods}

In this systematic review, an extensive search of published and unpublished randomised controlled trials (RCTs) of niacin, fibrates and CETPi's revealed 11 RCTs of niacin (35 301 participants), 20 of fibrates (46 099 participants) and 8 of CETPi's (36011 participants) with all-cause mortality as an outcome. These were subjected to meta-analysis. Secondary end points included coronary heart disease (CHD) mortality and non-fatal myocardial infarction (MI).

\section{Results}

None of the drug classes was associated with reduction in all-cause or CHD mortality. Niacin and fibrates were investigated both in patients receiving statins and those not on statins, whereas CETPi's were tested only against a background of statin therapy. Non-fatal MI occurred less frequently on niacin alone versus placebo (HR 0.69; 0.56 to 0.85, $\mathrm{p}<0.001, \mathrm{n}=4991$ ), but in combination with a statin it had no significant effect. Similarly, fibrates alone decreased the incidence of non-fatal MI (HR $0.78 ; 0.71$ to $0.86, \mathrm{p}<0.001, \mathrm{n}=32086$ ), but statistical significance was lost with statin co-administration.

\section{Commentary}

Keene and colleagues report that none of the three classes of drugs with HDL-raising properties decreased total or CHD mortality. Furthermore in recent trials, in which they were tested against a background of statin treatment, they even failed to reduce non-fatal MI. Non-fatal MI has a much higher incidence than fatal MI and thus arguments about inadequate statistical power cannot be applied as they can to fatal events. The findings complement another recent meta-analysis revealing that the increase in HDL cholesterol, which occurs with some statins, does not contribute any benefit beyond that from LDL reduction. ${ }^{2}$

Intriguingly, in the study by Keene and colleagues both niacin and fibrates did show significant decreases in non-fatal MI in trials in which statins were not co-prescribed. This is potentially important for patients with statin intolerance. In the case of niacin, however, it may be spurious, because the trials without statin were small and it is dubious whether they can properly be regarded as blinded due to the flushing reaction in the majority of participants randomised to active treatment. Laropiprant, which substantially reduces flushing, was not available until it had become unethical to conduct CVD end point trials in the absence of a statin. ${ }^{3}$ The fibrate findings are less easy to dismiss; numbers are substantial, fibrates well-tolerated and a similar trend for decreased nonfatal MI was present even when statins were co-prescribed. The question about why fibrates do not decrease all-cause mortality, however, remains worrying. ${ }^{4}$

It is too early to write-off CETPi's. Two large studies, one of evacetrapib (ACCELERATE (NCT01687998)) and another of anacetrapib (REVEAL (NCT01252953) have yet to report in 2016 and 2017, respectively.

A more sophisticated view of HDL is emerging; it has many functions beyond those of simple cholesterol carriage. It may not be rate limiting for reverse cholesterol transport, particularly when hepatic LDL uptake is stimulated by statins. The capacity of HDL to protect proteins from chemical modifications, such as oxidation and glycation, which do not relate to its cholesterol component, may be important in ameliorating atherogenesis and, because HDL is the most abundant lipoprotein in tissue fluid, perhaps other pathological processes. ${ }^{5}$

\section{Competing interests None.}

Provenance and peer review Commissioned; internally peer reviewed.

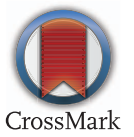

\section{References}

1. NICE. Clinical Guideline CG181. Lipid modification. Cardiovascular risk assessment and the modification of blood lipids for the primary and secondary prevention of cardiovascular disease. London: National Institute for Health and Care Excellence, 2014

2. Boekholdt SM, Arsenault BJ, Hovingh GK, et al. Levels and changes of HDL cholesterol and apolipoprotein A-I in relation to risk of cardiovascular events among statin-treated patients: a meta-analysis. Circulation 2013;128:1504-12.

3. HPS2-THRIVE Collaborative Group. Effects of extended release niacin with laropiprant in high risk patients. N Engl J Med 2014;371:203-12.

4. Oliver M. The clofibrate saga: a retrospective commentary. Br J Clin Pharmacol 2012;74:907-10.

5. Soran H, Hama S, Yadav R, et al. HDL functionality. Curr Opin Lipidol 2012;23:353-66. 